\title{
The role of interventional pain management in chronic pain
}

\author{
Alex Cahana ${ }^{1}$, Philippe Mavrocordatos ${ }^{1}$, Elisabeth Van Gessel ${ }^{1}$, and Setsuro Ogawa ${ }^{2}$ \\ ${ }^{1}$ Interventional Pain Program, Department of Anesthesiology, Pharmacology, Intensive Care, Geneva University Hospital, \\ 24 Rue Micheli-du-Crest, 1211 Geneva 14, Switzerland \\ ${ }^{2}$ Department of Anesthesiology, Surugadai Nihon University Hospital, Tokyo, Japan
}

Key words Back pain - Cancer pain - Interventional pain management

\section{Introduction}

Alleviating pain is not a recent concern. All know that intense chronic pain has a profound, distressing influence on a person's physical and mental wellbeing. Because pain is the most common disabling disease, it imposes severe economic stresses on the patient and society; thus, it constitutes serious national and world health problems. But it is only in treating the patient as a person, rather than as a passive receptacle, that the role of interventional pain management has begun to advance.

Interventional pain management offers a spectrum of minimally invasive procedures, with both diagnostic and therapeutic intent, for patients suffering from chronic, persistent, and intense pain. Patients with multiple back surgeries, endstage cancer, and neuropathic, ischemic, and central pain now have hope, and as we move into the twenty-first century with improved technologies borrowed from many disciplines, we are closing the gaps in our knowledge that were conferred by scientific ignorance. Neuromodulation, novel drugs with novel drug delivery systems, and even gene therapy are all out there on the horizon.

Deficiencies in pain management still exist, but pain medicine is rapidly approaching responsibility and recognition, and we hope that, in time, pain medicine will become an independent specialty. Nevertheless, the days of the omnipotent, omnipresent physician who "waves his hand" over a silent, awestruck patient are over.

Address correspondence to: A. Cahana

Received: August 20, 2003 / Accepted: November 4, 2003

\section{Conservative treatments and their limitations}

Acute pain, the subjective response to injury, is something we may be able to comprehend and accept as a necessary and even welcome signal, to avoid impending injury and similar encounters in the future. By contrast, chronic unrelenting pain, divorced from its essential protective context, becomes a meaningless burden that can wear down even the stoutest heart. There is nothing physical to learn from it, and in time, the sufferer is tired, and in desperation may seek "no matter what kind of treatment".

This desperation, shared by the patient as well as the physician, makes decision-making in pain management extremely difficult and at times emotional. Many factors profoundly influence this decision, including the physician's attitudes, beliefs, medical cognizance, and training; the patient's age, overall health, functional status, and ethno-cultural and religious beliefs, as well as preferences; and also regulatory forces, facilities, and economic factors [1].

Until recently, opioids were used for pain relief only in the most desperate of medical conditions, with a curious, but contextually understandable dichotomy towards pain of malignant versus nonmalignant origin [2]. Contemporary approaches to managing pain emphasize earlier and more liberal use of opioids, citing a low potential for addiction and an overall favorable riskbenefit ratio. However, while there is reasonable scientific support for this general contention, in practice, symptom control remains completely empirical.

The role of minimally invasive and anesthetic painrelieving modalities is even more ill defined, creating a situation analogous to that with opioids. Although there is fairly widespread agreement that procedures have a favorable risk-benefit ratio, no uniformly accepted validated methods have been devised to prospectively determine in what settings which procedures are valuable. 
Nevertheless, the use of interventional pain management techniques has grown exponentially, and with this has come a better understanding of what can and cannot be expected from these procedures. It is beyond the scope of this review to explain the multitude of minimally invasive procedures for all refractory pain syndromes; thus, we shall focus on the basics: patient selection, procedure selection, and outcomes known to date in the literature.

\section{Patient selection}

The cornerstone of patient selection is an accurate pain diagnosis, avoiding exclusion diagnoses such as fibromyalgia, psychogenic pain, somatization disorder, and malingering, while creating a bond of trust with the patient, which later will be essential in pursuing a therapeutic program. Patient selection criteria for most interventional procedures are shown in Table 1.

Confounding this already difficult picture is the presence of variables that may adversely directly or indirectly influence outcome. Motivation is the first and foremost important variable that affects procedure results. Patients with secondary gain issues, litigation, or a desire to be relieved of social responsibilities will probably not do well, even with a perfectly executed technique. Low compliance history with previously prescribed treatment regimens and chemical dependency on opioids, benzodiazepines, and barbiturates are notoriously known to be difficult to manage. Finally, there is a correlation between educational level and ultimate outcome, due in part to the presence of poor coping strategies, no return-to-work incentive, and lack of comprehension of procedure outcome [3]. Other negative predictors include the chronicity of pain, the presence of comorbidities such as diabetes, osteoporosis, peripheral vascular disease, and others, and poor operator skills.

Patient selection is best done by a team approach, thus addressing medical, interventional, psychological, and vocational aspects of the proposed therapeutic scheme. One must bear in mind that, with certain procedures (spinal cord stimulators, intrathecal pumps, epidural port-a-cath's, Deltec, St. Paul, MN, USA) therapeutic relationships between the patient and the

Table 1. Patient selection criteria

1. Pain refractory to all previous conservative treatments

2. Pain responsive, but accompanied by an intolerable sideeffect profile

3. Patient understands and accepts treatment

4. No coagulopathy or infection

5. No major psychopathology interventionist will persist, perhaps throughout life, and regular communication and surveillance is essential.

\section{Procedure selection}

\section{What to do with refractory back pain?}

Disabling low back pain is a significant and common medical problem in the Western world, affecting millions of people annually. Back pain is reported as the second leading symptom resulting in physician visits in the United States and Europe, and estimates of direct medical costs are staggering. Ninety percent of adults experience low back pain during their lifetime, although resolution of symptoms occurs with non-specific treatments in $75 \%-90 \%$ of patients. It is estimated that the remaining $10 \%$ of refractory patients account for $80 \%$ of the overall cost of back problems. In patients under the age of 45 , low back pain results in more disability than any other health problem, and overall is second only to the common cold in incidence [4].

Many guidelines exist regarding the treatment of low back pain [5]. Regardless of its etiology (e.g., herniated nucleus pulposus, internal disc disruption, degenerative disc disease, facet syndrome, myofascial pain syndrome, ligamentous strain, tumor, vertebral compression fractures, and spinal stenosis), it is clear that failure of conservative treatment for more than 4 weeks merits an interdisciplinary approach, which includes medical, surgical, interventional, and psychological analysis.

From an interventional point of view, there are several key questions that construct the rationale of the treatment algorithm (Table 2).

As we can see, "back pain" includes a multitude of different cases. A middle-aged patient suffering from low back pain after lifting a heavy load, without clear radicular signs or conclusive imaging findings, is different from a young patient suffering from persistent leg pain after two herniated disc operations associated with the presence of postoperative fibrosis, which is different from an elderly patient suffering from back and leg pain associated with activity, with diffuse degenerative changes shown on imaging.

Diagnosis is essential, and interventional pain techniques are minimally invasive procedures that have diagnostic as well as therapeutic value. They do not

Table 2. Key questions to be asked prior to treatment

1. Is the patient suffering from back pain? leg pain? both?

2. Is the pain mostly nociceptive? neuropathic? mixed?

3. Are there signs of true or pseudoradiculopathy?

4. Can the imaging findings explain the pain?

5. Has the back been operated upon? 


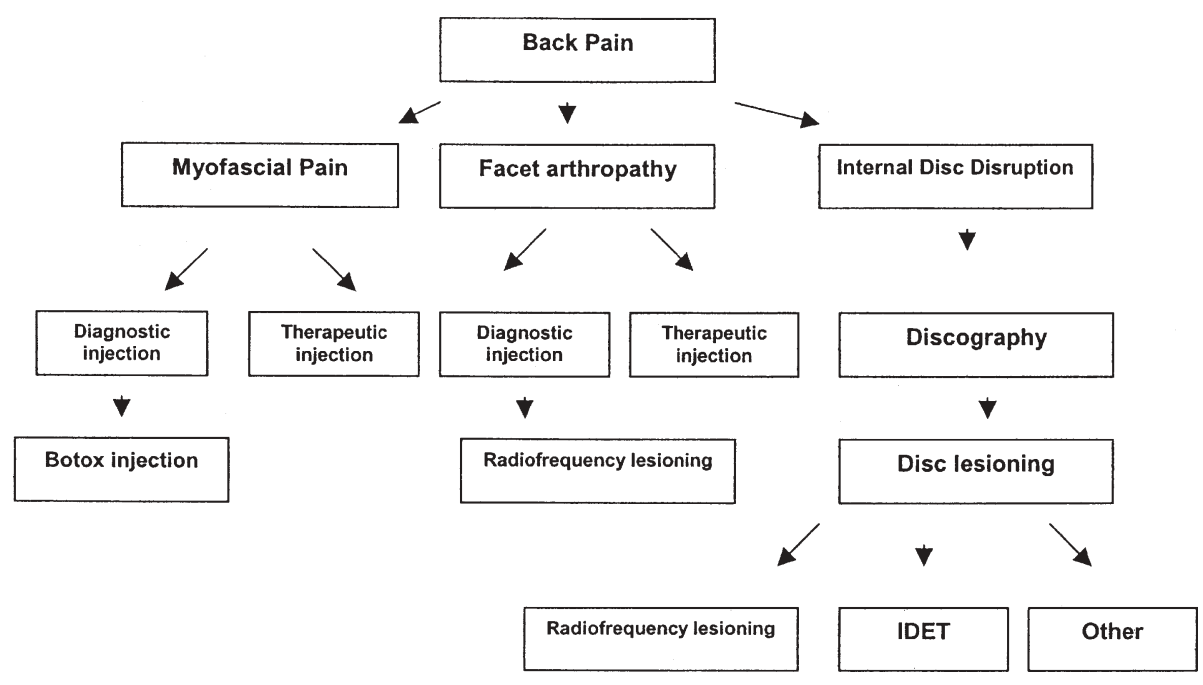

Fig. 1. Back pain algorithm. IDET, intradiscal electrotherapy replace surgery when indicated, or medical treatment when efficient. These are complementary procedures, which, on one hand, may increase the sensitivity and specificity of certain tests (e.g., selective nerve root blocks, discography, epiduroscopy), and they may also assist in persistent pain syndromes where medication side effects (mostly those of nonsteroidal antiinflammatory drugs [NSAIDS] and opioids) are unwarranted or intolerable (e.g., cortisone infiltration, radiofrequency $[\mathrm{RF}]$ lesioning).

When pain persists, despite an unrevealing workup, and inflammatory, metabolic, and tumor etiologies have been excluded one must determine whether this is back pain with or without painful radiculopathy (Fig. 1).

As one can see, back pain with no, or with pseudoradiculopathy usually emanates from three main etiologies. Pure facet arthropathy (also known as facet syndrome) is the sole etiology in only $15 \%-20 \%$ of chronic low-back-pain patients, but, when it exists, treatment by RF lesioning is efficacious [6]. The rationale for the application of RF denervation is the assumption that the selective heating of nervous structures can impede nociceptive input. Practically, this is achieved by the percutaneous application of a small-size electrode at target neural tissues, resulting in sizecontrolled lesions at different anatomical positions (Fig. 2).

RF lesioning is always preceded by two to three diagnostic blocks done under fluoroscopic control with a minute amount of local anesthetic to make sure that there is no placebo response to the procedure. If responses to diagnostic blocks are unequivocal, a definitive treatment is offered, with an effect that usually lasts between 12 and 18 months, and the treatment can be repeated regularly. Other sites, such as the sacroiliac joint, seem responsive to treatment, and RF lesioning offers a promising therapeutic alternative [7] (Fig. 3).

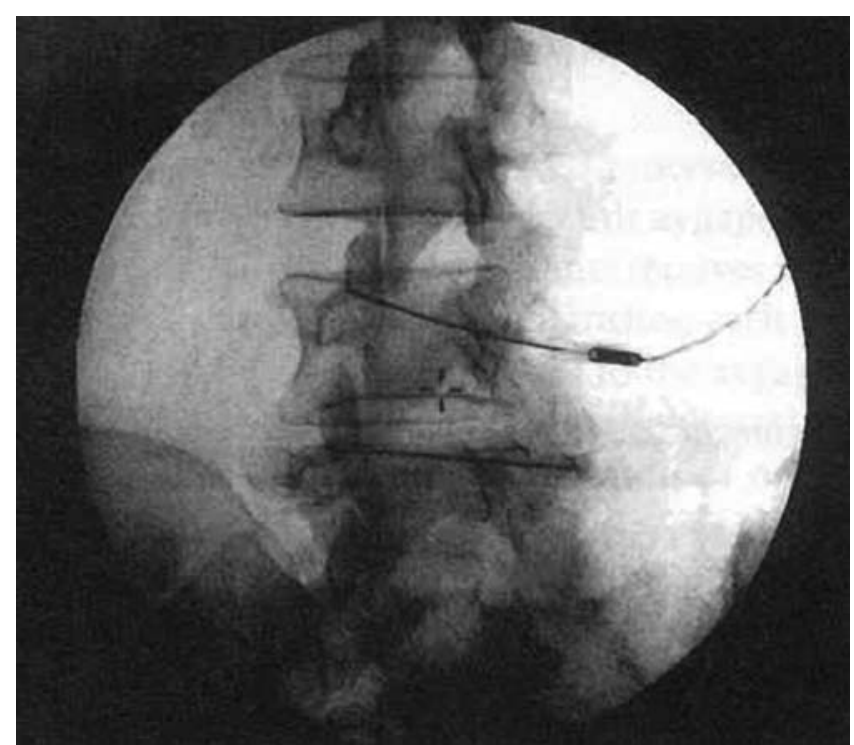

Fig. 2. Median branch block for the diagnosis of low back pain suspected to originate from the zygapophysial (facet) joint

Disc disease, however, is much more complex in nature and involves multiple pain targets, such as posterior annulus nociceptors, segmental dorsal root ganglions, and sympathetic chain and segmental nerve roots when the disc is compressed. It is therefore difficult to think of one "magical" infiltration in these cases, with an absolute therapeutic effect. However, in patients who are not candidates for surgery (no frank neural deficit, absence of sequestered disc, no segmental instability), RF lesioning of the disc may offer a therapeutic alternative [8].

Intradiscal electrotherapy (IDET) is a percutaneous procedure using RF as a heat source which induces collagen changes within the disc, which, in turn, can decrease disc protrusion, as well as producing nocicep- 


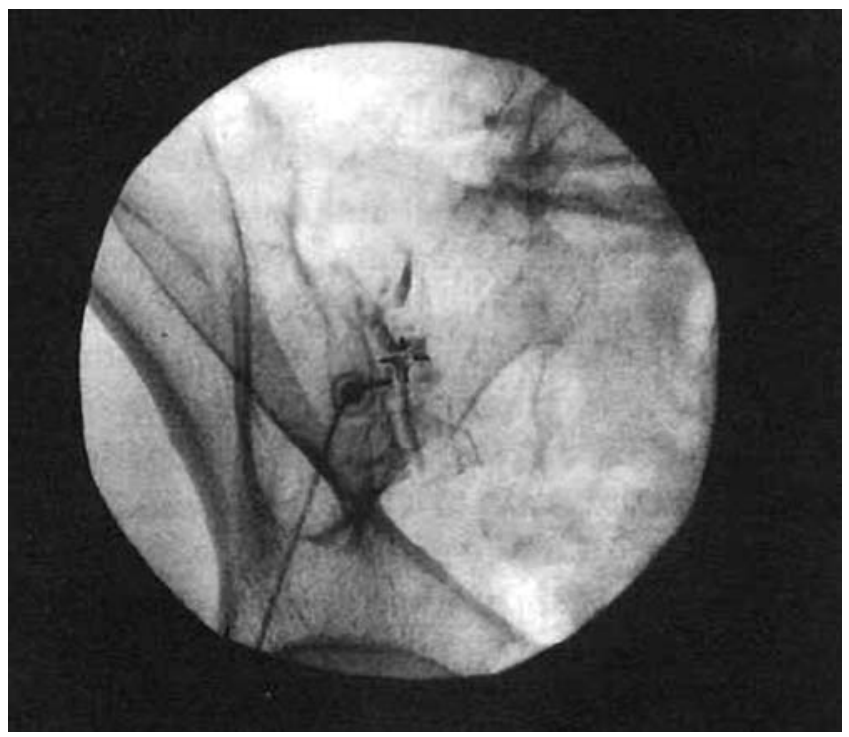

Fig. 3. Sacroiliac joint block for the diagnosis of low back pain suspected to originate from the sacroiliac joint

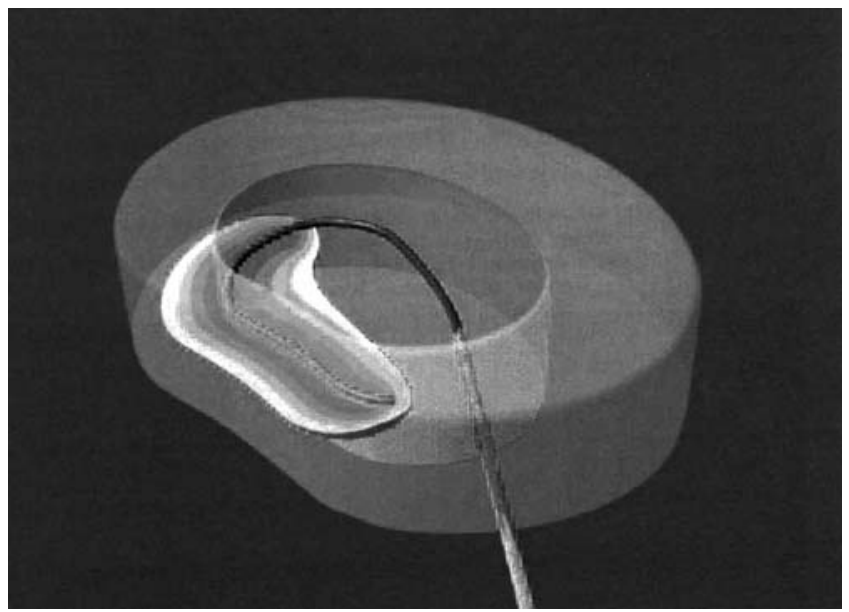

Fig. 4. Intradiscal electrotherapy (IDET) for the treatment of low back pain from a painful disc

tor denervation of the posterior annulus due to the heating effect. IDET is always preceded by a diagnostic discography, and its effects, after appropriate rehabilitation, last for years (Fig. 4).

When radicular pain is suspected, another approach is employed (Fig. 5).

In radicular pain it is essential to differentiate between operated backs (also known as failed back surgery syndrome [FBSS]), versus nonoperated backs, because a suspicion of the presence of epidural fibrosis alters the therapeutic yield of injections [9].

Conflicting studies over the past 40 years have been reported on the efficacy of lumbar epidural steroids in

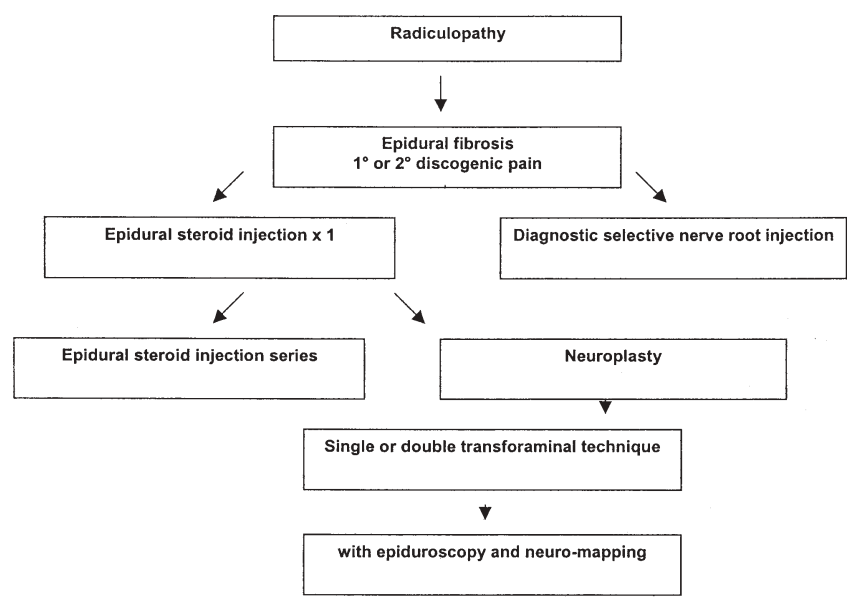

Fig. 5. Leg pain algorithm

patients with radicular pain [10]. Despite the controversies, these injections are frequently performed and most practitioners feel strongly that patients do derive a significant benefit. Yet, all acknowledge that some patients receiving steroid injections do not improve, perhaps due to poor patient selection, misdiagnosis, and inappropriately administered or misplaced injections.

If the effect of epidural steroid injections is local, i.e., a direct effect on the injured nerve root, then it is essential that the steroid reaches the site of injury. Historically, epidural steroid injections have been performed "blindly", without any radiological guidance. Many factors may prevent the steroid from reaching the intended nerve root, including scarring, adhesions, and epidural adipose tissue and septa, which may be present both in the patient who has undergone previous lumbar spine surgery and in the patient who has never had back surgery [11].

In the presence of epidural fibrosis, drugs injected will follow the path of least resistance. Theoretically, this will divert the drug away from the painful nerve associated with the fibrotic material. This supports the clinical observation that many patients report significant relief after one injection but not after another. It is thus essential that any instillation of medication be sitespecific under fluoroscopic control.

Epidural neuroplasty (also known as the Racz procedure) [12] consists of accessing the epidural space in the caudal or transforaminal approach, and injecting nonionic contrast material (thus performing an epidurogram) in order to detect "filling defects". This is followed by the gentle manipulation of a metal reinforced catheter in order to liberate adhesions ("filling the defects"), and then by the injection of the targeted medication. This procedure, which allows prolonged pain relief in refractory cases, has the advantage of targeted drug delivery, but has the disadvantage of indi- 


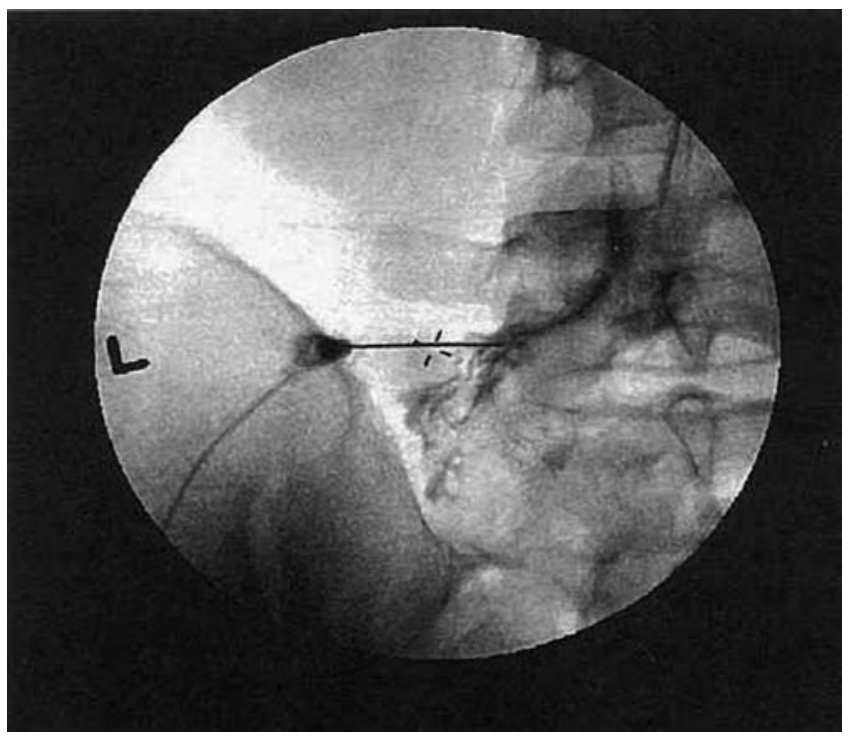

Fig. 6. Percutaneous neuroplasty for the treatment of persistent low back and leg pain from a nerve root fibrosis

rect, two-dimensional vision of the presumed pathology (Fig. 6).

With the advent of the use of endoscopic technology in pain medicine, epiduroscopy has been introduced. In essence, this procedure includes the insertion of a fine fiberoptic endoscope via the caudal hiatus, under fluoroscopic control, to the presumed level of pathology. Epidural structures and nerve roots are directly visualized, and the presence of inflammation and fibrosis is noted. The procedure is done under local anesthesia, while continuously monitoring the intra-epidural pressure, and the patient's response. Normal nerve roots, when touched, cause paresthesia, but diseased ones hurt, so the patient's report is essential while the operator gently performs adhesiolysis. Recent results are promising [13], and the future association with intraoperative nerve stimulating is exciting.

When radiculopathy is refractory or without the clear presence of fibrosis, a second approach may be used (Fig. 7).

Again, selective nerve root injections that are either diagnostic or therapeutic in nature are warranted; however, a more imaginative approach must be used.

Neuropathic pain is usually considered to be a contraindication for the use of RF lesioning, because it makes little sense to perform a neurodestructive procedure in the presence of altered neural function, as this risks aggravating the neural pathology (i.e., deafferentation pain, neural damage). However, pulsed RF (PRF), where short bursts of RF energy are applied to the nerve, is thought to be a safer alternative to classical $\mathrm{RF}$, and to date there is no clinical evidence of neural damage [14]. The mechanism by which PRF works re-

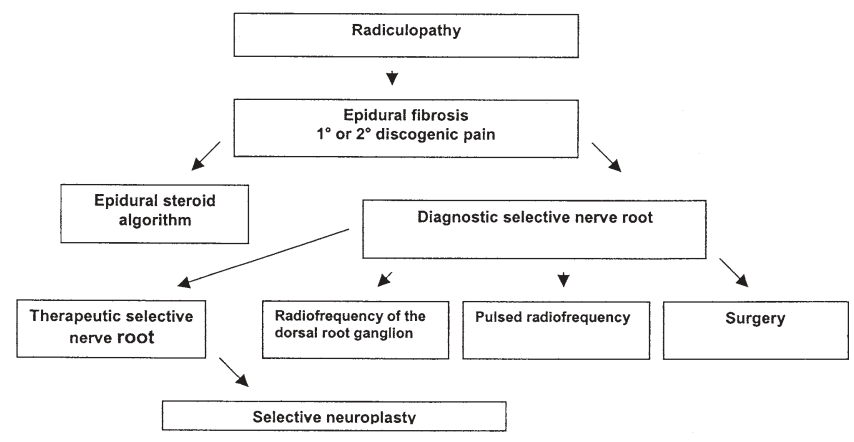

Fig. 7. Refractory leg pain algorithm

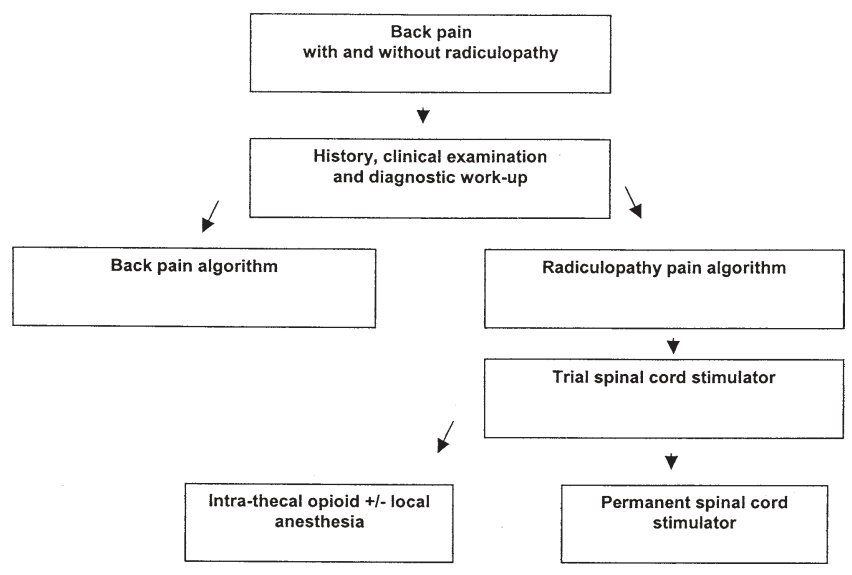

Fig. 8. Implantable device algorithm

mains unclear. Some studies suggest that the analgesic effect is a neuromodulatory rather than a neurodestructive one [15].

PRF is basically performed exactly like thermal RF, with an apparatus capable of transmitting the appropriate energy. The field of PRF opens new horizons to other treatments of neuropathic pain syndrome, such as postherpetic neuralgia, frozen shoulder, intercostal neuralgia, complex regional pain syndrome (CRPS) type I, and more.

Finally, "when all else failed" and the pain persists, the utilization of implantable devices (such as spinal cord stimulators and implantable pumps) comes into place (Fig. 8).

\section{What to do with refractory cancer pain?}

Surprisingly enough, the treatment of refractory cancer pain is much simpler and more straightforward than the treatment of back pain. Perhaps because the etiologies of cancer pain are less enigmatic than those of back pain, perhaps we feel more comfortable in introducing invasive procedures in dire situations, or perhaps we feel ethically obliged "to do something" in cancer pain, 


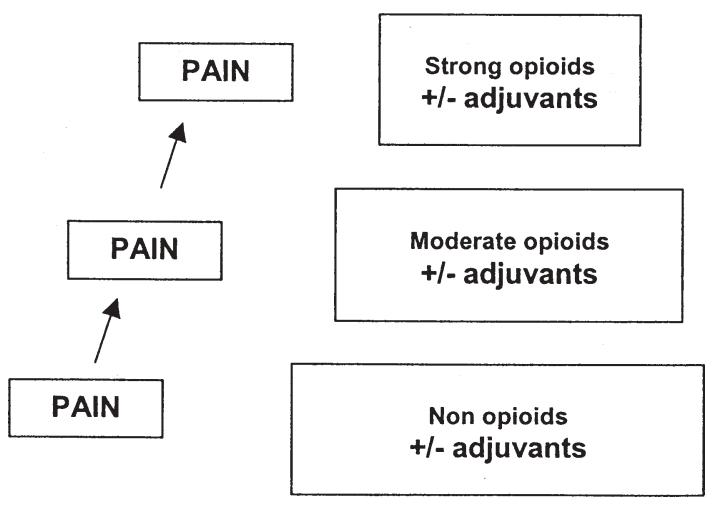

Fig. 9. WHO analgesic ladder

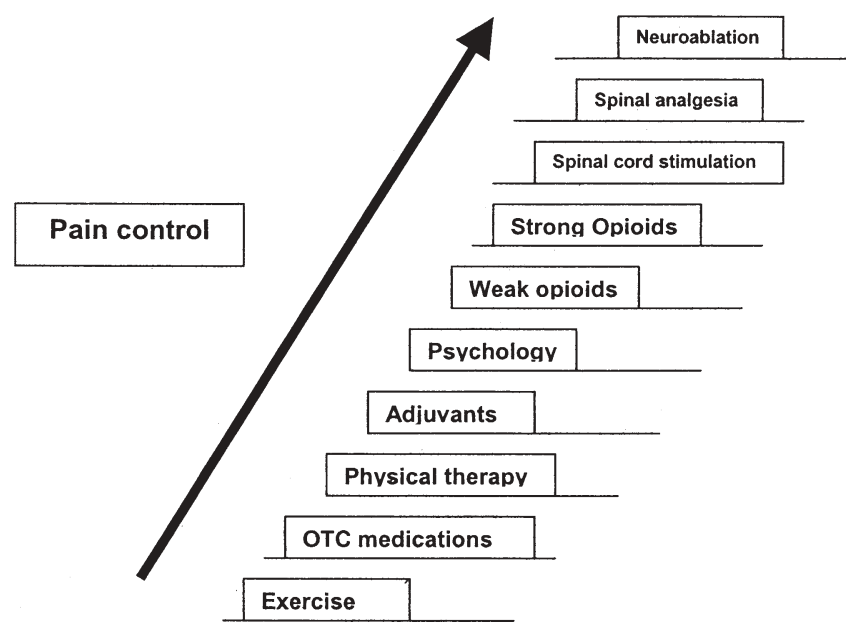

Fig. 10. Pain control continuum. $O T C$, over-the-counter

which is regarded as a more "acceptable and understood" type of pain.

Eighty to ninety percent of cancer patients respond to the WHO analgesic ladder (Fig. 9) when it is appropriately used [16]. However, $10 \%-20 \%$ do not respond. This is not a negligible number, and it is imperative that physicians do not give up seeking alternative paincontrol techniques. All too often physicians rely only on familiar therapies, even when these fail to control pain, and patients pass the last weeks of their lives in unnecessary suffering. This need not be.

Available therapies include epidural or intrathecal drug delivery systems (such as port-a-cath, or completely internalized systems), neurolytic (also known as neuroablative) procedures (such as alcohol neurolysis), and neurosurgical procedures (e.g., cordotomy, myelotomy) (Fig. 10).

Whatever the treatment, the patient selection criteria remain the same (Table 1). Decision on implanting an external versus internal, or an intrathecal versus epidural system depends on the patient's general status, life

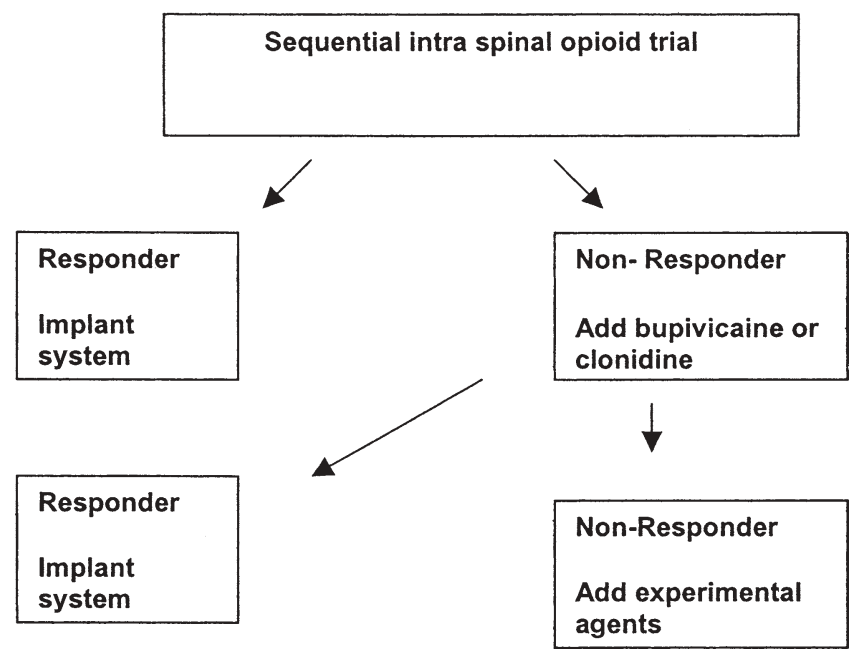

Fig. 11. Pump implantation algorithm

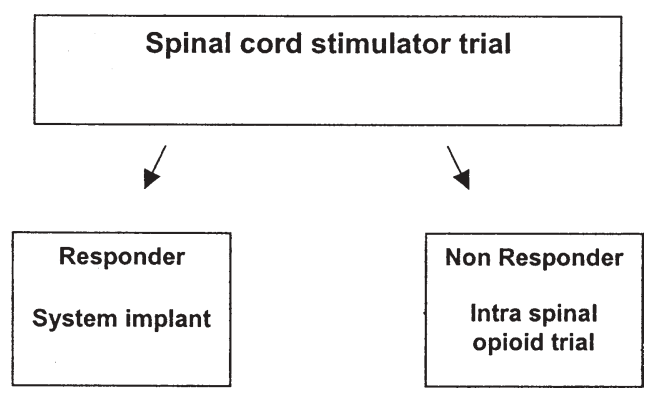

Fig. 12. Spinal cord stimulator algorithm

expectancy, and technical support availability [17]. Roughly speaking, patients with thoraco-abdominal or lower limb pain with a life expectancy of between 3 and 12 months will do well with an epidural port-a-cath system, while patients with metastatic diffuse pain should have either partially or completely internalized intrathecal systems.

All implantations are preceded by a diagnostic trial, and those who are responsive are considered for a definitive treatment (Fig. 11). Different medications (mostly morphine) have been proposed.

As for spinal cord stimulation, it is used as a neuromodulatory tool in cases of refractory neuropathic pain (CRPS type I, phantom pain, postherpetic neuralgia); ischemic pain (refractory angina, peripheral vascular disease); mixed pain (failed back surgery syndrome); or cancer pain $[18,19]$. The implantation of temporary electrodes in the epidural space precedes the implantation of a permanent generator (Fig. 12). A trial period, varying between 3 days and 3 weeks, discloses the patient's response to this treatment. Once decided upon, the pacemaker is internalized in the abdominal wall (usually the left lower quadrant) or the buttocks, 
and further follow-up is similar to that of a patient with a cardiac pacemaker.

\section{Conclusions}

It is important to remember that all medical interventions are associated with risks and benefits, which compromise the famous risk-benefit ratio. All interventions have alternatives (including no intervention), and each alternative possesses its own risk-benefit ratio. Thus, clinical decision-making involves comparing and contrasting the risk-benefit ratio not only of the procedure, but of the alternative intervention as well.

In this short review we have not discussed many other important interventional modalities, such as cryo-analgesia, vertebroplasty, and kyphoplasty, and various somatic and sympathetic blocks. It is not our aim to recall an exhaustive list of theoretical procedures, but, rather, to remind us that pain medicine in general, and interventional pain medicine in particular, have recently emerged as a lively and dynamic subspecialty, with ever-changing techniques and approaches developing before our very eyes.

Debate over the role of invasive procedures for chronic pain will probably rest for a while, evoking strong emotions similar to those evoked during the "opioid phobia" era in the 1980s. Be that as it may, there is an agreement that outcomes for procedures are most salutary when they are properly integrated into a multidisciplinary matrix. But this contention demands collaboration, collegial discussion, and praise for innovation and open horizons. Evidence-based medicine is but a tool to drive us to create our own evidence and not to rest passively back and wait for it to come.

Our moral obligation dictates not just "to do no harm", but to do "good" as well [20].

\section{References}

1. Minzter BH, Cahana A (2000) Organizing an inpatient pain service. In: Raj PP (ed) Practical management of pain. Mosby, St. Louis, pp 17-38

2. Passik SD, Portenoy RK, Ricketts PL (1998) Substance abuse issues in cancer patients part 1: prevalence and diagnosis. Oncology 12:517-521
3. Borenstein DG (2000) Epidemiology, etiology, diagnostic evaluation and treatment of low back pain. Curr Opin Rheumatol 12:143-149

4. Andersson GB (1999) Epidemiological features of low back pain. Lancet 354:581-585

5. Koes BW, van Tulder MW, Ostelo R, Kim Burton A, Waddell G (2001) Clinical guidelines for the management of low back pain in primary care: an international comparison Spine 26:2504-2513

6. Geurts JW, van Wijk RW, Stolker RJ, Groen GJ (2001) Efficacy of radiofrequency procedures for the treatment of spinal pain: a systematic review of randomized clinical trials. Reg Anesth Pain Med 26:394-400

7. Ferrante FM, King LF, Roche EA, Kim PS, Aranda M, Delaney LR, Mardini IA, Mannes AJ (2001) Radiofrequency sacroiliac joint denervation for sacroiliac syndrome. Reg Anesth Pain Med 26:137-142

8. Saal JS, Saal JA (2002) Intradiscal electrothermal treatment for chronic discogenic low back pain: prospective outcome study with a minimum 2-year follow-up. Spine 27:966-973

9. Anderson SR (2000) A rationale for the treatment of failed back surgery syndrome. Curr Pain Headache Rep 4:395-406

10. Machikanti L, Staats PS, Singh V, Schultz DM, Vilims BD, Jasper JF, Kloth DS, Trescot AM, Hansen HC, Falasca TD, Racz GB, Deer TR, Burton AW, Helm S, Lou L, Bakhit CE, Dunbar EE, Atluri SL, Calodney AK, Hassenbuch SJ, Feler CA (2003) Evidence based practice guidelines for interventional techniques in the management of chronic spinal pain. Pain Physician 6:381

11. Mulligan KA, Rowlingson JC (2001) Epidural steroids. Curr Pain Headache Rep 5:495-502

12. Heavner JE, Racz GB, Raj P (1999) Percutaneous epidural neuroplasty: prospective evaluation of $0.9 \% \mathrm{NaCl}$ versus $10 \%$ $\mathrm{NaCl}$ with or without hyaluronidase. Reg Anesth Pain Med 24:202-207

13. Geurts JW, Kalleward JW, Richardson J, Groen GJ (2002) Targeted methylprednisolone acetate/hyaluronidase/clonidine injection after diagnostic epiduroscopy for chronic sciatica: a prospective 1 year follow-up study. Reg Anesth Pain Med 27:343352

14. Munglani R (1999) The longer term effect of pulsed radiofrequency for neuropathic pain. Pain 80:437-439

15. Cahana A, Vutskits L, Muller D (2003) Acute differential modulation of synaptic transmission and cell survival during exposure to pulsed and continuous radiofrequency energy. J Pain 4:197-202

16. Stjernsward J, Colleau S, Ventafridda V (1996) The WHO cancer pain and palliative care program. Past, present, and future. J Pain Symptom Manage 12:65-72

17. Lordon SP (2002) Interventional approach to cancer pain. Curr Pain Headache Rep 6:202-206

18. Deer TR (2001) Current and future trends in spinal cord stimulation for chronic pain. Curr Pain Headache Rep 5:503-509

19. Burchiel KJ, Anderson VC, Brown FD, Fessler RG, Friedman WA, Pelofsky S, Weiner RL, Oakley J, Shatin D (1996) Prospective, multicenter study of spinal cord stimulation for relief of chronic back and extremity pain. Spine 21:2786-2794

20. Cahana A (2002) Is optimal pain relief always optimal? Bioethical considerations of interventional pain at the end of life. APS Bulletin 12:3-6 